\title{
The Gouy phase of Airy beams
}

\author{
Xiaoyan Pang, ${ }^{1}$ Greg Gbur, ${ }^{2}$ and Taco D. Visser ${ }^{1,3, *}$ \\ ${ }^{1}$ Faculty of Electrical Engineering, Mathematics and Computer Science, Delft University of Technology, Delft, The Netherlands \\ ${ }^{2}$ Department of Physics and Optical Science, University of North Carolina at Charlotte, 9201 University City Boulevard, \\ Charlotte, North Carolina 28223, USA \\ ${ }^{3}$ Department of Physics and Astronomy, and Institute for Lasers, Life and Biophotonics, VU University, Amsterdam, The Netherlands \\ *Corresponding author: T.D.Visser@tudelft.nl
}

Received April 8, 2011; accepted May 26, 2011;

posted May 27, 2011 (Doc. ID 145643); published June 23, 2011

\begin{abstract}
The phase behavior of Airy beams is studied, and their Gouy phase is defined. Analytic expressions for the idealized, infinite-energy type beam are derived. They are shown to be excellent approximations for finite-energy beams generated under typical experimental conditions. (C) 2011 Optical Society of America

OCIS codes: $\quad 050.1960,350.5030$.
\end{abstract}

Beams that do not spread on propagation, so-called nondiffracting beams, have attracted considerable attention since they were discovered by Durnin et al. [1-3]. A special type of such beams are the Airy beams described by Berry and Balazs in the context of quantum mechanics [4]. These beams have the remarkable property that they "accelerate" away from the original direction of propagation. Airy beams are idealizations, because they carry an infinite amount of energy. Siviloglou and Christodoulides discussed how an exponentially modulated Airy function source would produce a finite-energy beam, which would retain its nondiffracting and accelerating behavior over an appreciable propagation distance [5]. After the experimental realization of such a beam [6], several studies have been devoted to their properties [7-10], and a number of applications are being pursued. For instance, the "self-healing" capacity of Airy beams [11] makes them excellent candidates for optical communication through turbulent media [12]. Other intriguing applications are the generation of curved plasma channels [13], and the manipulation of particles along bends in labs-on-achip [14].

Traditionally, the term Gouy phase describes how the phase of a monochromatic, focused field differs from that of a plane wave with the same frequency (see [15] and the references therein). Recently, however, it has also been used to describe the phase of a nondiffracting Bessel beam [16]. In this Letter we study the phase behavior of both finite-energy and infinite-energy Airy beams. By comparing their phase to that of a suitable reference field, their Gouy phase can be defined. A good understanding of the phase properties of Airy beams is of importance in interferometric or remote-sensing applications employing them.

Consider a monochromatic, one-dimensional beamlike wave field $U(x, z, \omega)$ that propagates in the positive $z$ direction, and can be written as

$$
U(x, \boldsymbol{z}, \omega)=\phi(x, \boldsymbol{z}) e^{\mathrm{i}(k z-\omega t)},
$$

with the envelope $\phi(x, z)$ a solution of the paraxial wave equation

$$
\frac{\partial^{2} \phi}{\partial x^{2}}+2 i k \frac{\partial \phi}{\partial z}=0
$$

Here $k=\omega / c$ is the wavenumber associated with frequency $\omega, c$ denotes the speed of light, and $t$ the time. A possible solution of Eq. (2) is the so-called Airy beam, given by the expression [4]

$$
\phi(s, \xi)=\mathrm{Ai}\left[s-\left(\frac{\xi^{2}}{4}\right)\right] \exp \left[i\left(\frac{s \xi}{2}-\frac{\xi^{3}}{12}\right)\right],
$$

with Ai the Airy function, $s=x / x_{0}$ a dimensionless transverse coordinate, and $\xi=z / k x_{0}^{2}$ a normalized propagation distance. In the remainder the constant $x_{0}$ is taken to be positive, and the time-dependent part of the wave field is suppressed. An example of the intensity distribution of an Airy beam is shown in Fig. 1, from which both the diffraction-free propagation and the transverse acceleration can be seen.

Because of its curved trajectory, we define the Gouy phase $\delta$ of an Airy beam as the difference between its phase $\psi$ and that of an ideal (nondiffracted) diverging cylindrical wave $U_{\mathrm{cyl}}(x, z, \omega)$ centered on the $y$-axis and propagating into the half-space $z>0$, i.e.,

$$
\delta(x, z, \omega)=\psi[U(x, z, \omega)]-\psi\left[U_{\mathrm{cyl}}(x, z, \omega)\right],
$$

with

$$
U_{\text {cyl }}(x, z, \omega)=\frac{i C}{4} H_{0}^{(1)}(k \rho) .
$$

Here $C$ is a complex-valued constant, $H_{0}^{(1)}$ denotes a Hankel function of the first kind of order zero, and

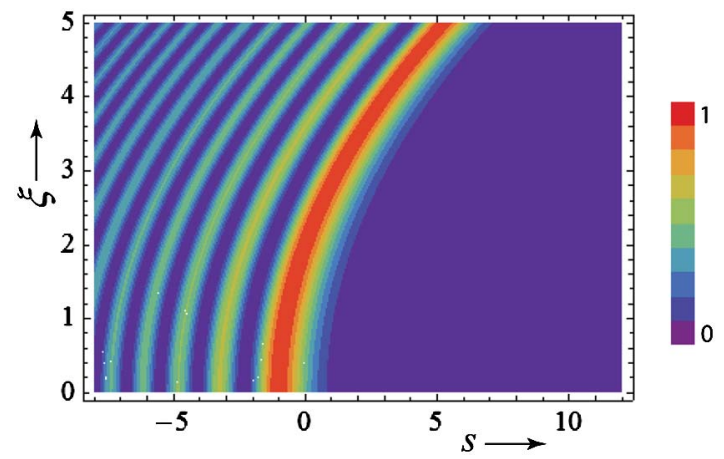

Fig. 1. (Color online) Normalized intensity distribution of an Airy beam propagating in the positive $\xi$-direction. 
$\rho=\left(x^{2}+z^{2}\right)^{1 / 2}$. The asymptotic behavior of the cylindrical wave field is given by the expression

$$
U_{\text {cyl }}(x, z, \omega) \sim C \sqrt{\frac{2}{\pi k \rho}} e^{i(k \rho-\pi / 4)}, \quad(k \rho \gg 1 / 4) .
$$

We choose the constant $C$ in Eq. (5) such that $\psi\left[U_{\text {cyl }}(x, z, \omega)\right]=k \rho$. For $z \gg x$ this may be written as

$$
k \rho \approx k z\left[1+\frac{1}{2}\left(\frac{x}{z}\right)^{2}\right]=k z+\frac{1}{2} \frac{s^{2}}{\xi} .
$$

Thus, we have from Eqs. (1 $)$, (3), and (ㅁ) that

$$
\delta(s, \xi, \omega)=\frac{s \xi}{2}-\frac{\xi^{3}}{12}-\frac{s^{2}}{2 \xi}+\psi_{\mathrm{Ai}},
$$

where $\psi_{\mathrm{Ai}}$ is the phase of the Airy function of Eq. (3). For real values of its argument the Airy function is real, and hence $\psi_{\mathrm{Ai}}$ equals 0 or $\pi$. The first zero of $\operatorname{Ai}(x)$ occurs near $x=-2.34$. On making use of this in Eq. (3), we find that $\psi_{\mathrm{Ai}}=0$ when $\xi<2(s+2.34)^{1 / 2}$. We first restrict our attention to this region of $s \xi$-space.

It is seen from Eq. (3) that the maximum beam intensity, $|\phi(s, \xi)|^{2}$, occurs on a quadratic trajectory. We therefore study the behavior of the Gouy phase on curves of the type $s=\alpha \xi^{2}$, with $a$ a positive constant. On substituting this form into Eq. (8), it immediately follows that the Gouy phase vanishes identically along two curves, viz.

$$
\delta(s, \xi, \omega)=0, \quad \text { if } s=\left(3 \pm 3^{1 / 2}\right) \xi^{2} / 6 .
$$

Similarly, it is seen that the maximum Gouy phase occurs along the curve $s=\xi^{2} / 2$, namely

$$
\delta(s, \xi, \omega)=\frac{\xi^{3}}{24}, \quad \text { if } s=\xi^{2} / 2 .
$$

The quadratic trajectory along which the intensity equals $\mathrm{Ai}^{2}(0)$ (next to the maximum intensity, see Fig. 1), is given by the expression $s=\xi^{2} / 4$. On substituting this form into Eq. (ㅇ) we find that

$$
\delta(s, \xi, \omega)=\frac{\xi^{3}}{96}, \quad \text { if } s=\xi^{2} / 4 .
$$

We notice in passing that along the $\xi$-axis (i.e., the $z$ direction) the Gouy phase takes on negative values, i.e.,

$$
\delta(0, \xi, \omega)=-\frac{\xi^{3}}{12} .
$$

Contours of the Gouy phase are shown in Fig. 2. Superposed are several quadratic curves. It is seen that the two dashed curves given by Eq. (9) indeed coincide with the zero contours. The curve along which the Gouy phase reaches its maximum [see Eq. (10)] is displayed as a solid line. The dotted curve is given by Eq. (11).

We next turn our attention to the region $\xi>2(s+2.34)^{1 / 2}$. Here the Airy function can take on the value zero. At such points its phase $\psi_{\mathrm{Ai}}$ is singular,

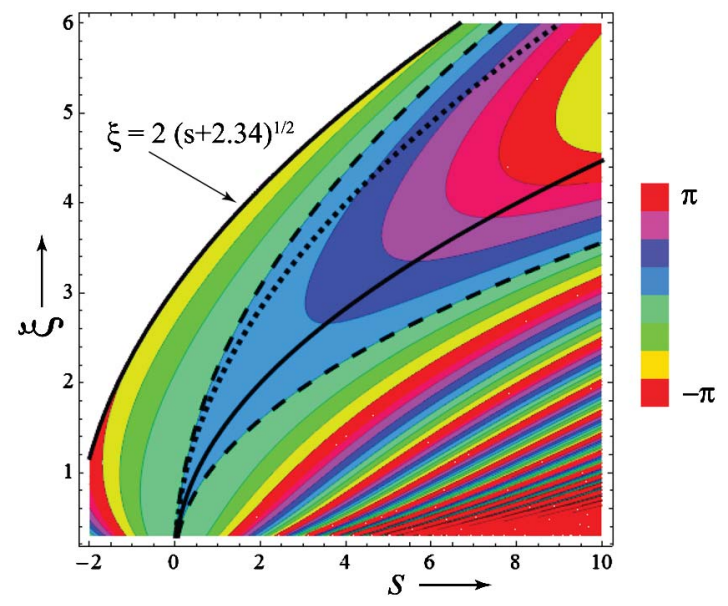

Fig. 2. (Color online) Color-coded plot of the Gouy phase of an Airy beam. Only the $s \xi$-region in which the Airy function has no zeros is shown. Along the two dashed curves, given by Eq. (9), the Gouy phase equals zero. Along the solid curve, given by Eq. (10), the Gouy phase reaches its maximum. The dotted curve is given by Eq. (11).

as is the Gouy phase. Both phases display a discontinuity of an amount $\pi$ at these singularities. An example of this behavior is shown in Fig. 3. The diagonal line that runs from the left-hand bottom to the right-hand top indicates the fifth zero of the Airy function, i.e., $\operatorname{Ai}\left(s-\xi^{2} / 4=\right.$ $-7.94)=0$. It is seen from the color-coding that the Gouy phase exhibits a $\pi$-discontinuity across this line.

The beams we discussed so far are idealizations because the Airy function is not square integrable, i.e., a beam described by Eq. (3) carries an infinite amount of energy. Siviloglou and Christodoulides [ㄷ] considered an Airy beam source with an exponential envelope, i.e.,

$$
\phi^{(\mathrm{fe})}(s, 0)=\operatorname{Ai}(s) e^{a s}
$$

with the decay parameter $a>0$ as to ensure a finiteenergy contribution, called ( $f e$ ), from the tail of the Airy function. They showed that such a beam propagates as

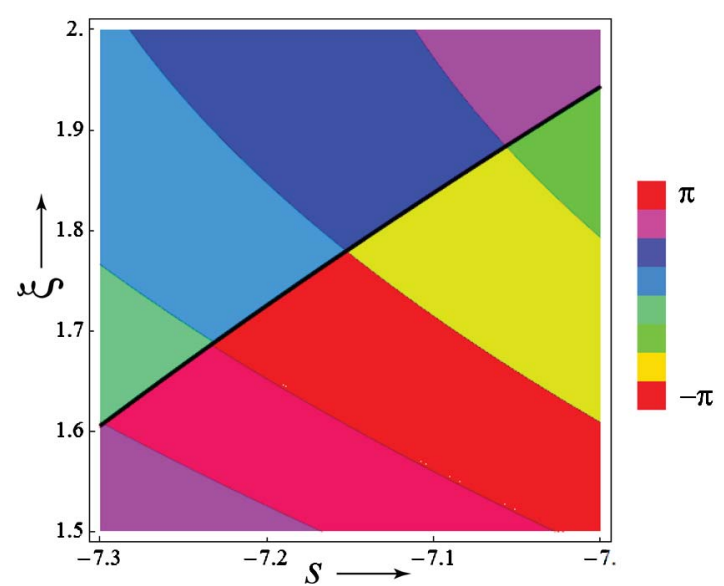

Fig. 3. (Color online) Color-coded plot of the Gouy phase of an Airy beam. A portion of the region in which the function $\operatorname{Ai}(x)$ has zeros is shown. The solid black line indicates the fifth zero of the Airy function. The Gouy phase jumps by an amount $\pi$ across this line. 


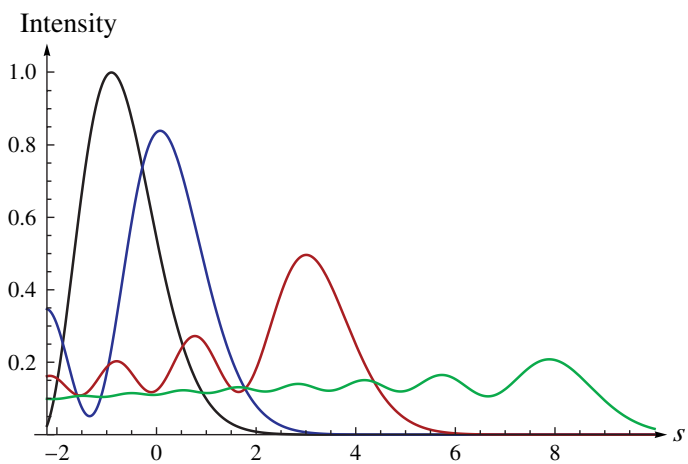

Fig. 4. (Color online) Intensity of a finite-energy Airy beam in different cross sections perpendicular to the $\xi$-axis: the source plane $\xi=0$ (black), $\xi=2$ (blue), $\xi=4$ (red), and $\xi=6$ (green).

$$
\begin{aligned}
\phi^{(\mathrm{fe})}(s, \xi)= & \operatorname{Ai}\left(s-\xi^{2} / 4+\mathrm{i} a \xi\right) e^{a s-a \xi^{2} / 2} \\
& \times e^{\left[\mathrm{i}\left(-\xi^{3} / 12+a^{2} \xi / 2+s \xi / 2\right)\right]}
\end{aligned}
$$

Such a finite-energy beam still shows the characteristic acceleration and is, at least to some extent, diffraction-free. A beam of this type has been realized using a Gaussian beam incident on a spatial light modulator [6]. It follows from Eqs. (4) and (14) that the Gouy phase for such beams is given by the expression

$$
\delta^{(\mathrm{fe})}(s, \xi, \omega)=\frac{s \xi}{2}-\frac{\xi^{3}}{12}-\frac{s^{2}}{2 \xi}+\frac{a^{2} \xi}{2}+\psi_{\mathrm{Ai}}
$$

It is to be noted that $\psi_{\mathrm{Ai}}$ now pertains to the Airy function of Eq. (14), and is no longer restricted to the values 0 and $\pi$. In the experiment reported in [6], the parameter values were $x_{0}=53 \mu \mathrm{m}, a=0.11$, and $\lambda=488 \mathrm{~nm}$. In Fig. 4 , selected cross sections of the corresponding beam intensity are shown. On propagation the height of the central peak gradually decreases and the beam remains essentially diffraction-free up to $\xi \approx 5$ (corresponding to a propagation length of $18 \mathrm{~cm}$ ), after which it rapidly spreads. However, the result expressed in Eq. (9), namely that the Gouy phase is zero along two quadratic curves, is still an excellent approximation under these conditions. This is shown in Fig. 5 in which the Gouy phase $\delta^{(\mathrm{fe})}(s, \xi, \omega)$ is plotted along the curves $s=\left(3 \pm 3^{1 / 2}\right) \xi^{2} / 6$. It is seen that the actual value of the phase anomaly is always less than 2 . This corresponds to a deviation of less than $\lambda / 3$ from the approximate value zero after a propagation distance of 360,000 wavelengths. Along the curves of Eqs. (10) and (11) the difference between the analytic expressions pertaining to the infinite-energy beam and a numerical evaluation of Eq. (15) is even smaller.

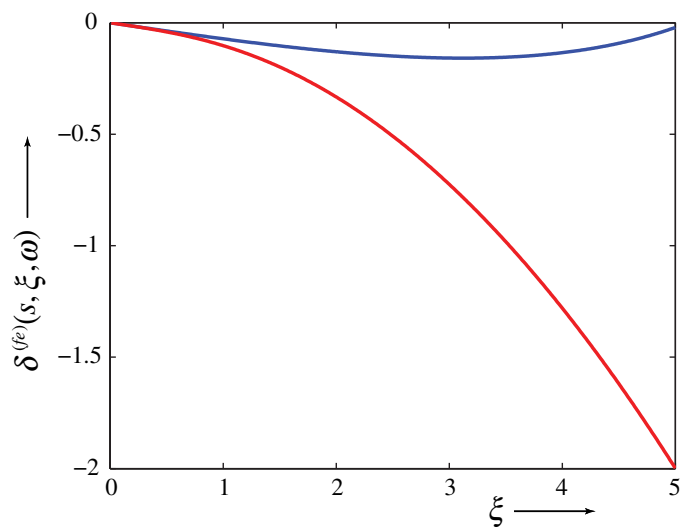

Fig. 5. (Color online) Gouy phase of a finite-energy Airy beam along the curves $\left.s=\left(3+3^{1 / 2}\right) / 6\right] \xi^{2}$ (red), and $s=$ $\left.\left(3-3^{1 / 2}\right) / 6\right] \xi^{2}$ (blue).

In conclusion, the phase behavior of infinite-energy Airy beams has been analyzed. By comparing this behavior to that of an outgoing cylindrical wave, analytic expressions for their Gouy phase were derived. It was shown numerically that these results are excellent approximations for the Gouy phase of finite-energy Airy beams generated under typical conditions.

\section{References}

1. J. Durnin, J. Opt. Soc. Am. A 4, 651 (1987).

2. J. Durnin, J. Miceli, and J. H. Eberly, Phys. Rev. Lett. 58, 1499 (1987).

3. J. Turunen and A. T. Friberg, in Progress in Optics, Vol. 54, E. Wolf, ed. (Elsevier, 2010), pp. 1-88.

4. M. V. Berry and N. L. Balazs, Am. J. Phys. 47, 264 (1979).

5. G. A. Siviloglou and D. N. Christodoulides, Opt. Lett. 32, 979 (2007).

6. G. A. Siviloglou, J. Broky, A. Dogariu, and D. N. Christodoulides, Phys. Rev. Lett. 99, 213901 (2007).

7. M. A. Bandres, Opt. Lett. 33, 1678 (2008).

8. J. E. Morris, M. Mazilu, J. Baumgartl, T. Cizmar, and K. Dholakia, Opt. Express 17, 13236 (2009).

9. S. Vo, K. Fuerschbach, K. P. Thompson, M. A. Alonso, and J. P. Rolland, J. Opt. Soc. Am. A 27, 2574 (2010).

10. Y. Kaganovsky and E. Heyman, Opt. Express 18, 8440 (2010).

11. J. Broky, G. A. Siviloglou, A. Dogariu, and D. N. Christodoulides, Opt. Express 16, 12880 (2008).

12. Y. L. Gu and G. Gbur, Opt. Lett. 35, 3456 (2010).

13. P. Polynkin, M. Kolesik, J. V. Moloney, G. A. Siviloglou, and D. N. Christodoulides, Science 324, 229 (2009).

14. J. Baumgartl, G. M. Hannappel, D. J. Stevenson, D. Day, M. $\mathrm{Gu}$, and K. Dholakia, Lab Chip 9, 1334 (2009).

15. T. D. Visser and E. Wolf, Opt. Commun. 283, 3371 (2010).

16. P. Martelli, M. Tacca, A. Gatto, G. Moneta, and M. Martinelli, Opt. Express 18, 7108 (2010). 